\title{
Orientar la asignatura de Anatomía y Fisiología hacia las competencias profesionales de enfermería. Propuesta de un proyecto docente.
}

\author{
Orienting the subjects of anatomy and physiology to nursing professional \\ competence. A teaching proposal.
}

Anna Falcó Pegueroles

Escola Universitària d'Infermeria Gimbernat (adscrito a la UAB). Sant Cugat del Vallès (Barcelona).

El estudio de la anatomía y la físiología del cuerpo humano forma parte de las materias básicas e imprescindibles en la formación de los profesionales de la salud. Sin embargo, las competencias profesionales de la enfermería actual, el entorno sanitario, y la interrelación con otras materias del currículum, establecen unas necesidades de formación muy específicas en el campo de la anatomía, la fisiología, y la bioquímica. En la actualidad, muchos de los proyectos docentes relativos a estas materias que se imparten en las Escuelas universitarias de Enfermería están diseñados a partir de un modelo tradicional que introduce con éxito al alumno en los conceptos anatómicos y fisiológicos más importantes y fundamentales del cuerpo humano, sin duda, pero no permite a éste integrarlos con el resto de asignaturas ni asegurar el aprendizaje de contenidos útiles y necesarios desde un punto de vista práctico. El presente artículo analiza los factores que explican este desajuste entre los contenidos de la asignatura de anatomía y fisiología y las necesidades de formación; también expone un proyecto docente que orienta los
Correspondencia:

Anna Falcó Pegueroles

EUI Gimbernat

Avinguda de la Generalitat s/n.

08174 Sant Cugat del Vallès. (Barcelona)

e-mail: anna.falco@cesc.es contenidos y los métodos pedagógicos a estas exigencias de formación en enfermería; y busca compartir con otros docentes la experiencia de creación y ejecución de un proyecto de estas características en un momento de transformación académica con motivo de la integración del sistema universitario español al espacio europeo de enseñanza superior.

Palabras clave: educación basada en competencias, proyecto docente, anatomía, fisiología, enfermería, enseñanza universitaria.

The study of anatomy and physiology of the human body is an essential part in the education of all health professionals. However, the professional competence in today's nursing, the health environment, and their interrelation with other subjects in the nursing curriculum require very specific educational needs in the field of anatomy, physiology, and biochemistry. Nowadays, most of the teaching projects on this subject at nursing university schools are designed following a traditional model. This successfully introduces the most important and basic anatomical and physiological concepts to the nursing student but it neither facilitates their integration with other subjects nor ensures the learning of useful and necessary concepts from a practical point of view. This article analyses the factors that account for this lack of adjustments between the syllabus of anatomy and physiology and educational needs. It also presents an educational project that orientates the contents and the pedagogical methods used to this demands in the nursing field. The study also tries to share with other teachers the experience 
in the creation of a project of these characteristics in a period of academic changes brought by the integration into the European higher education landscape.

Key words: competence-based education, outcome-orientededucation, teaching project, anatomy, physiology, nursing, University teaching.

\section{INTRODUCCIÓN}

El estudio de las estructuras orgánicas y sistemas del cuerpo humano, y la comprensión de sus funciones, forma parte de las materias consideradas básicas e imprescindibles en la formación de los profesionales de la salud. Enfermeras, fisioterapeutas, podólogos, odontólogos, entre otros, y naturalmente, médicos, tienen en sus currícula formación específica en áreas de conocimiento de anatomía, fisiología, bioquímica y física. A pesar de este denominador común de la materia en las diferentes disciplinas de la salud, las competencias profesionales definen en cada caso necesidades distintas a la hora de establecer los contenidos mínimos de éstas áreas del saber. Como afirma Arias ${ }^{1}$, se debe reconocer que no son iguales las competencias de las diversas profesiones en ciencias de la salud y que las necesidades de formación general entre titulaciones pueden ser diferentes.

En Enfermería las materias de anatomía, fisiología y bioquímica forman parte del currículum desde los inicios preuniversitarios. Con el tiempo y las diversas reformas de los planes de estudio, los contenidos relativos a estas disciplinas han ido sufriendo modificaciones en relación, básicamente, a la carga lectiva. Este hecho ha conducido inevitablemente a una reestructuración de los temarios a impartir pero no ha significado, en la mayoría de los casos, una reorientación verdadera de estos contenidos hacia las necesidades de formación de enfermería.

Las competencias profesionales de la enfermería actual, el entorno sanitario, y la interrelación con otras materias del currículum, establecen unos requerimientos de formación muy específicos en el campo de la anatomía, la fisiología, y la bioquímica.

El presente artículo expone un proyecto docente de la asignatura de Estructura y función del cuerpo humano, troncal de primer curso de la Diplomatura de Enfermería, que orienta los contenidos y los métodos pedagógicos a las necesidades de formación de la diplomatura, y busca compartir con otros docentes la experiencia de creación y ejecución de un proyecto de estas características.

\section{DESAJUSTES ENTRE CONTENIDOS Y NECESIDADES DE FORMACIÓN}

Algunas investigaciones ${ }^{1}$ sobre los contenidos de determinadas materias en los planes de estudio de diplomaturas de ciencias de la salud han evidenciado que la condición de algunas asignaturas troncales, como sería la anatomía y la fisiología humana, no mencionan aspectos específicos de la carrera en que se estudian ni permiten identificar ésta.

En la actualidad, muchos de los proyectos docentes relativos a las materias de anatomía y fisiología que se imparten en las Escuelas universitarias de Enfermería están diseñados a partir de un modelo tradicional que introduce al estudiante en los conceptos anatómicos y fisiológicos del cuerpo humano más importantes y fundamentales, sin duda, pero no aseguran el aprendizaje de contenidos verdaderamente útiles para el desarrollo profesional real. Es decir, el alumno recibe una buena formación desde una perspectiva médico-clínica relativa a estas materias pero no acaba de relacionar ni integrar los contenidos con el resto de asignaturas ni con su experiencia práctica. Este hecho plantea la pregunta si una enfermera debe dominar conceptos relevantes de anatomía y fisiología que no aplicará, con toda probabilidad, en un entorno profesional.

El porqué de este desajuste entre los contenidos y las necesidades de formación puede explicarse por varios factores. El primero es que la asignatura la imparte, en la mayoría de los casos, un Licenciado en Medicina, un profesional experto en las materias de anatomía y fisiología, pero desconocedor del ámbito competencial de la enfermería y del resto de asignaturas del currículum formativo de la Diplomatura.

Otro factor, es la falta de interrelación con otras asignaturas del currículum de enfermería. Como anteriormente se apuntaba, el profesor de anatomía y fisiología desconoce con frecuencia el contenido e interrelaciones entre las diferentes asignaturas troncales y obligatorias. Esto provoca en la mayoría de casos, una clara desconexión entre los contenidos que se imparten en primer curso, en la asignatura de anatomía y fisiología, y los necesarios para iniciar, por ejemplo, enfermería medico-quirúrgica, farmacología, o procedimientos específicos realizados en clases prácticas o en estancias hospitalarias. 
Un tercer factor, es la disminución lectiva que ha sufrido la asignatura en las últimas reformas de los planes de estudios. El profesor ha diseñado los programas a partir de modelos tradicionales que potencian los contenidos generalistas en el campo de la anatomía y la fisiología, y ha ido readaptando estos contenidos a las nuevas exigencias. Sin embargo, en muchos casos, esta readaptación no ha ido acompañada de una orientación de los temarios hacia las competencias profesionales de enfermería.

Otra variable relacionada con el desajuste entre los contenidos y las necesidades de formación que ha propiciado un modelo tradicional de los proyectos docentes de anatomía y fisiología que se imparten en la actualidad, es la aplicación de una metodología fundamentada en las clases magistrales o expositivas, no potenciando el rol activo del alumno, limitándose éste a tomar apuntes y, en algunos casos, a ampliarlos con la bibliografía recomendada por el profesor. Como afirma Prada ${ }^{2}$, tradicionalmente los contenidos teóricos se enseñan a través de clases magistrales a pesar que una buena enseñanza implica comprometer a los estudiantes en el proceso de aprendizaje. La introducción de metodologías como las clases prácticas, seminarios o simulaciones que permiten al alumno percibir la comunicación no verbal del profesor favorece la adquisición rápida del contenido enseñado ${ }^{3}$. Sin embargo, es necesario señalar que las horas lectivas planificadas, la carga docente y el volumen de alumnos por profesor son elementos determinantes para poder introducir estas metodologías más participativas.

Figura 1. Relación de la asignatura de Estructura y función del cuerpo humano con el resto de materias del currículum formativo de Enfermería.

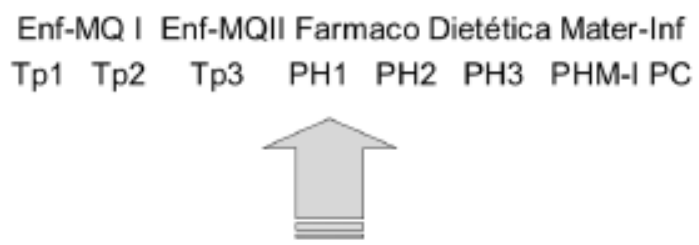

Estructura y función del cuerpo humano

Leyenda: Enf-MQ I: Enfermeria Medico-quirürgica I; Enf-MQ II: Enfermeria Medico-quirúrgica II; Farmaco: Farmacologia; Mater-Inf: Enfermeria Materno-Infantil; Tp1/2/3: Teorico-prácticas; PH: prácticas hospitalarias; PHM-I; Prácticas hospitalarias materno-infantil; PC: prácticas comunitaria.

\section{PROPUESTA DE UN PROYECTO DOCENTE}

Se diseña un proyecto docente que pretende orientar la asignatura de anatomía y fisiología hacia las competencias profesionales de enfermería. El punto de partida es el modelo de educación basado en las competencias finales del estudiante, un modo de enseñanza determinado por las necesidades de la sociedad ${ }^{4}$. Se realiza también una apuesta por la necesaria renovación de los contenidos de estas materias, además de potenciar la interrelación con otras asignaturas del currículum (Figura 1).

La idea que desarrolla este proyecto docente surge a partir de la formulación de una pregunta: ¿qué contenidos necesita saber y aplicar una enfermera generalista en relación a la anatomía y la fisiología humana en su ámbito de trabajo?. Se parte, pues, de las necesidades sociales y profesionales para orientar la definición de las competencias del estudiante, y a través de los objetivos de aprendizaje, son éstas las que inspiran el programa, los objetivos docentes y los criterios de evaluación (Figura 2).

Figura 2. Diseños tradicional y actual en el proceso de enseñanza-aprendizaje para algunas asignaturas que configuran el currículum de enfermería (de Falcó A. La nueva formación de profesionales: sobre la competencia profesional y la competencia del estudiante de enfermería. Educación Médica 2004;7(1):42-45.)

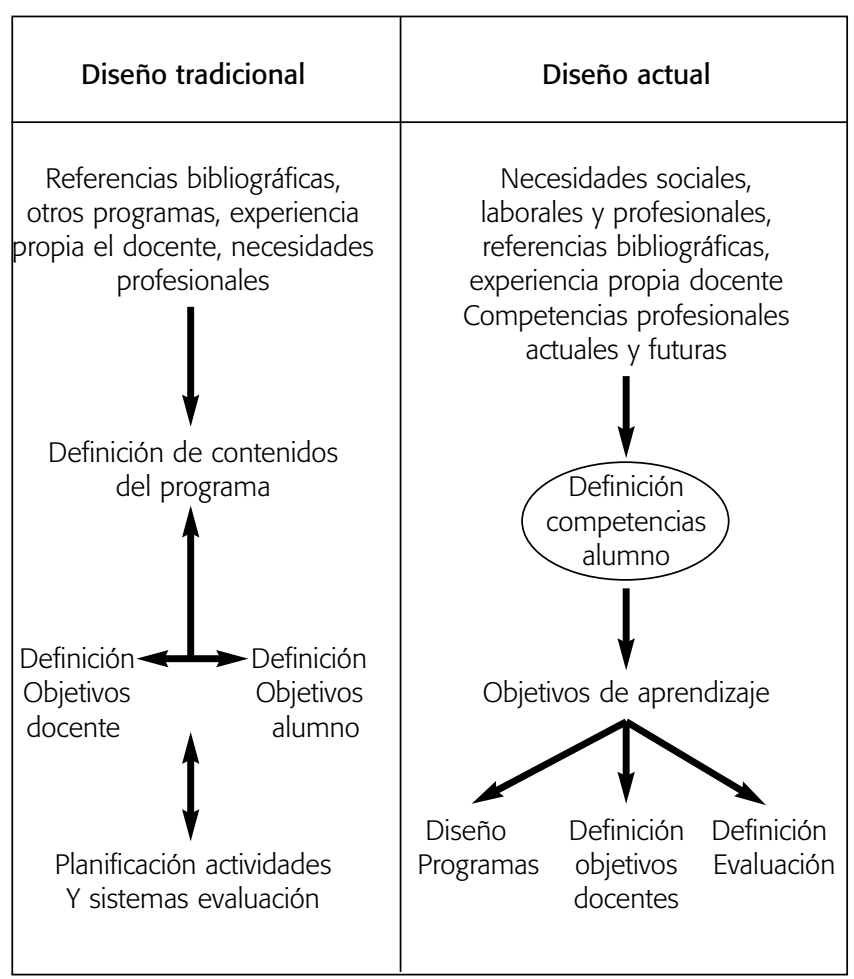


Se pone de manifiesto, así, la consideración de las competencias profesionales como el principal componente que ayuda a enfocar los contenidos, en este caso relativos a anatomía y fisiología en enfermería, hacia las necesidades reales de formación ${ }^{5}$, y hacia la definición de las competencias del estudiante. Como afirma Nolla ${ }^{6}$ esta definición debe presidir la construcción y el desarrollo curricular, dando luz a contenidos, metodología docente y estrategias educativas.

La asignatura de Estructura y función del cuerpo humano, troncal de primer curso, tiene por objetivos básicos introducir al alumno en el conocimiento de la anatomía y la fisiología del cuerpo humano, y asegurar el aprendizaje de contenidos previos necesarios para estudiar patología y cuidados de enfermería, en segundo y tercer curso. El proyecto docente presentado se desarrolla en cuatro unidades temáticas. Las dos primeras tratan los contenidos relativos a las bases de bioquímica, anatomía y fisiología. Son dos unidades fundamentales que justifican sus contenidos no sólo por la razón de ser de la asignatura, sino también porque pretenden consolidar conceptos que serán ampliados en temarios posteriores. Se ha pretendido optimizar los contenidos y buscar fórmulas para impartirlos de manera correcta, como son entregar a los alumnos apuntes, objetivos de aprendizaje y referencias bibliográficas para que trabajen fuera las aulas determinados conceptos.

La tercera unidad temática destaca por desarrollar los parámetros básicos hemodinámicos y los principales procesos internos de mantenimiento del equilibrio homeostático. Pretenden introducir el estudio en profundidad de los mecanismos generadores y de equilibrio de parámetros como la temperatura, la tensión arterial, la frecuencia cardíaca, la frecuencia respiratoria, el equilibrio hídrico y electrolítico y ácido-base.; además de asegurar el conocimiento, por ejemplo, de la sangre, las características de la piel y su regeneración, el metabolismo, o la comprensión de la exploración y auscultación básica del paciente. El aprendizaje de estos conceptos es fundamental para el estudiante de primer curso ya que sienta las bases del conocimiento de aspectos que abordará en el estudio de la patología, y en la mayoría de los procedimientos que la enfermera realiza o participa en la praxis asistencial.

Por último, la unidad temática práctica pretende aplicar conocimientos y desarrollar diferentes habilidades en el estudiante relacionadas de manera directa con los contenidos de las otras tres unidades; también lo introduce en metodologías de análisis y estrategias de razonamiento deductivo. Se busca la implicación del alumno en su aprendizaje y su responsabilidad a la hora de autoevaluarse.

Se detallan a continuación los contenidos formales y estructurales que configuran el proyecto docente que se presenta.

\section{Descripción de la asignatura:}

Nombre: Estructura y función del cuerpo humano.

Asignatura troncal de 10 créditos, de los cuales 8 son teóricos y 2 prácticos. Anual.

\section{Objetivos generales de la asignatura:}

- Introducir al alumno en el conocimiento de las partes de esqueleto humano y de sus órganos a partir de modelos anatómicos y material audiovisual.

- Proporcionar al alumno las bases de fisiología de los órganos y sistemas del cuerpo humano, los dispositivos de equilibrio orgánicos y los mecanismos para la integración de los conceptos y términos básicos en anatomía y fisiología.

- Analizar la fisiología y sus alteraciones básicas a partir de casos que simulan la realidad cotidiana.

- Optimizar y reforzar el conocimiento de los conceptos de anatomía y fisiología básicos y necesarios para el futuro profesional de enfermería.

\section{Temario general:}

Tema preliminar: El origen de la vida:

de la materia a la vida celular.

Unidad temática I: Estudio de las moléculas

y las macromoléculas

Tema 1: Bioelementos y biomoléculas

Tema 2: El agua y las sales minerales

Tema 3: Glúcidos.

Tema 4: Lípidos.

Tema 5: Proteínas.

Tema 6: Nucleoproteïnas.

Tema 7: Coenzimas y vitaminas.

Tema 8: Enzimas.

Tema 9: Hormonas.

Unidad Temática II: Organización y función del cuerpo humano

Tema 1: Bases conceptuales de anatomía y fisiología.

Tema 2: Génesis embrionaria de los tejidos. Clasificación y características de los tejidos.

Subunidad temática I. Estructura y movimiento. Subunidad temática II. Pensamiento y control de las actividades vitales. 
Subunidad temática III. Circulación

y respiración.

Subunidad temática IV. Digestión y excreción.

Subunidad temática V. Eliminación urinaria.

Subunidad temática VI. Reproducción humana.

Unidad temática III: Estudio de sistemas orgánicos

Subunidad temática I. El equilibrio

hidroelectrolítico y acido-base del organismo.

Subunidad temática II. La obtención de energía y el control de la glucemia.

Subunidad temática III. El estudio de la sangre y la inmunidad humana.

Subunidad temática IV. La actividad eléctrica del corazón y su registro.

Subunidad temática V. La presión sanguínea.

Fórmulas para su mantenimiento.

Subunidad temática VI. La temperatura y la termorregulación del cuerpo.

Subunidad temática VII. El tejido cutáneo y su estructura. Fases de regeneración.

Subunidad temáticaVIII. Las hormonas

y su comportamiento: introducción a la fisiología

del sistema endocrino.

\section{Unidad temática IV: Unidad práctica}

Seminario 1: Sistema musculoesquelético

I: Anatomía palpatoria $1^{a}$ parte. Cráneo, tronco, extremidades.

Seminario 2: Sistema musculoesquelético

II: Anatomía palpatoria 2 parte. Cráneo, tronco, extremidades.

Seminario 3: Exploración neurológica y

de los sentidos.

Seminario 4: Fisiología cardio-respiratoria.

Principales vasos arteriales y venosos, accesos venosos, pulsos y la tensión arterial

Seminario 5: Técnicas y métodos de exploración y auscultación torácica y abdominal.

Seminario 6: El balance hídrico.

Seminario 7: El equilibrio ácido-base y la

gasometría arterial

Seminario 8: Aprendizaje Basado en problemas

$(\mathrm{ABP})^{*}$ Introducción a la etiología fisio-patológica del síndrome vasovagal. Introducción a

la metodología del ABP.

Sessió 8: ABP

Sessió 9: ABP

Sessió 10: ABP

\section{Objetivos específicos de las unidades temáticas:}

Unidad temática I: Estudio de las moléculas

y las macromoléculas

- Introducir el alumno en el conocimiento de las estructuras moleculares y unidades funcionales del organismo.

- Definir los niveles básicos moleculares y macromoleculares de la vida.

- Facilitar la integración de los conceptos relativos a los comportamientos moleculares, macromoleculares y celulares, estructura y funciones.

\section{Unidad Temática II: Organización y función}

del cuerpo humano

- Introducir el alumno en el conocimiento del esqueleto humano y los órganos a partir de modelos anatómicos.

- Proporcionar las bases de fisiología de los órganos y sistemas del cuerpo humano.

- Establecer los mecanismos para la integración de los conceptos y términos utilizados en anatomía y fisiología.

- Analizar la físiología y sus alteraciones a partir de ejemplos y situaciones clínicas.

- Optimizar los conceptos de anatomía y fisiología básicos y necesarios para el futuro profesional de enfermería

\section{Unidad temática III: Estudio de sistemas}

orgánicos

- Explicar la base fisiológica de parámetros y sistemas orgánicos.

- Desarrollar los principales conceptos de fisiología básicos y necesarios para el futuro profesional de enfermería.

- Interrelacionar signos y síntomas a partir de la fisiología.

- Estudiar la fisiología a partir de ejemplos y casos clínicos.

- Reforzar los conocimientos considerados imprescindibles para el estudio posterior de la patología, y la comprensión de las medidas terapéuticas e instrumentales.

Unidad temática IV: Unidad práctica

- Aplicar los conocimientos adquiridos en las clases a partir de la experimentación y la resolución de casos.

- Desarrollar habilidades en la exploración y auscultación básica del cuerpo humano. 
- Estimular la participación y implicación del alumno en su aprendizaje.

- Introducir el alumno en el aprendizaje basado en problemas (ABP), potenciando las capacidades de trabajo grupal, búsqueda y análisis, crítica $\mathrm{y}$ exposición.

- Estimular el trabajo en grupo y la responsabilidad que genera.

- Introducir estrategias del razonamiento hipotético-deductivo.

\section{Metodología}

Los recursos metodológicos necesarios para desarrollar las diferentes unidades temáticas son: clases expositivas o magistrales; ejercicios y estudio de casos; análisis de material audiovisual; aprendizaje basado en problemas; tutorías individualizadas y/o grupales; seminarios en grupos de 15-20 miembros por profesor con modelos anatómicos y otro material.

\section{Temporalización}

El temario de la asignatura de Estructura y función del cuerpo humano se distribuye entre los dos semestres, con un total de $82^{* *}$ sesiones (Figura 3 ).

Las sesiones son de 1 hora y 30 minutos de duración. Para desarrollar los contenidos de manera correcta, es necesario realizar un total de 124 horas aproximadamente.

No se incluye en este cómputo general las horas destinadas a tutorías, corrección de ejercicios y trabajos, ni el tiempo de dedicación del alumno a preparar la asignatura fuera de las aulas.

\section{Material}

El material necesario para las clases expositivas consta de: ordenador, video, retroproyector, material

Figura 3. Distribución de sesiones por unidades temáticas

\begin{tabular}{|l|c|c|}
\hline \multicolumn{1}{|c|}{ Unidad temática } & Sesiones & \multirow{2}{*}{ Créditos } \\
\hline Unidad temática I & 10 & \multirow{2}{*}{8} \\
\cline { 1 - 2 } Unidad temática II & 26 & \\
\hline Unidad temática III & 16 & \multirow{2}{*}{2} \\
\hline Unidad temática IV & $\begin{array}{c}30 \\
\text { (10 sesiones por grupo) }\end{array}$ & \\
\hline
\end{tabular}

audiovisual, fichas-dibujos, ejercicios y apuntes diseñados por la profesora, objetivos de aprendizaje y relación de la bibliografía y internetgrafía recomendada.

Para la realización de los seminarios prácticos se requiere una aula condicionada que simule el entorno asistencial, con posibilidad de utilizar, entre otros, el siguiente material: modelos anatómicos del cuerpo humano, de órganos y de estructuras corporales; esfingonomanómetros, fonendoscopios, camillas y tallas de ropa, linternas.

\section{Evaluación}

Se diseña un examen escrito, con preguntas test, preguntas a completar, interpretación de imágenes, y preguntas a desarrollar.

La parte práctica se evalúa a partir de los criterios de asistencia, grado de conocimientos y participación del alumno.

La distribución de la nota es la siguiente:

Examen escrito: $80 \%$

Parte practica: $20 \%$ a añadir en el caso que la nota del examen sea igual o superior a 4 sobre 10 .

\section{CONCLUSIONES}

El proceso de cambio por el que pasan las universidades españolas en la actualidad, con motivo de la implantación de los criterios para integrar el sistema universitario español en el espacio europeo de enseñanza superior, ha generado un debate interno: ¿está la universidad formando profesionales suficientemente preparados para trabajar en el mundo laboral y social actual?.

Este debate ha introducido nuevas perspectivas educativas y ha ayudado a redefinir y concretar los conceptos de competencia profesional y competencias del estudiante, con el fin que las universidades titulen a personas no sólo por demostrar unos determinados conocimientos, sino también a partir de la demostración de actitudes y valores propios de la profesión a desarrollar. Dirigir la universidad hacia las actuales necesidades sociales implica la participación activa de todos los agentes que intervienen en la formación profesional en pregrado y posgrado, entre los cuales se encuentra el profesor universitario.

El docente debe aprovechar este momento de rea-

\footnotetext{
**En el curso académico 2004/05 en el que se inicia la implantación del proyecto docente, se planifican un total de 70 sesiones, puesto que no se realiza el Aprendizaje Basado en Problemas ni determina sesión magistral, supliéndola por la entrega al alumno de material realizado por el profesor correspondiente a los contenidos no trabajados en el aula. Se prevé para cursos posteriores poder realizar el total de sesiones necesarias, si la planificación y los recursos lo permiten.
} 
daptación universitaria para plantearse si los contenidos que imparte están en relación a las verdaderas necesidades de formación de los estudiantes. Aunque este planteamiento pasa ineludiblemente por la consideración de los recursos que le facilita la institución universitaria, es necesaria también la autocrítica y el intercambio. Ser autocrítico con el proyecto docente desarrollado ha de permitir la readaptación de los contenidos y las metodologías a las nuevas necesidades de formación. Intercambiar con otros docentes las ideas y reflexiones surgidas de la crítica ayuda a redefinir y concretar los conceptos de competencia profesional y competencias del estudiante para poder aplicarlos en las diferentes materias del currículum. Así se optimizan también los contenidos y se evita la frecuente repetición de temas en diferentes asignaturas y cursos.

El proyecto docente que se presenta surge de este análisis y pretende demostrar la orientación de los contenidos de anatomía y fisiología a las competencias profesionales de enfermería. El punto de partida, tal y como se ha expuesto con anterioridad, es el ámbito competencial de la enfermera: cuidar a la persona enferma y ayudar a las personas a desarrollar los cuidados de manera adecuada a sus necesidades.

Entre otras muchas tareas, para poder cuidar es necesario valorar parámetros hemodinámicos $\mathrm{y}$ antropométricos; detectar, interpretar y actuar frente a los signos y síntomas que se presentan; y trabajar conjuntamente con el médico para unir cura y cuidado. Así pues, si se pretende que el estudiante domine, entre otras, estas habilidades propias de la profesión, se considera imprescindible destinar tiempo y recursos al aprendizaje de la fisiología y sus alteraciones, conceptos que se desarrollan en diversas asignaturas del currículum: Estructura y función del cuerpo humano, Enfermería Medico-quirúrgica, Farmacología o Dietética, entre otras; y en los distintos cursos académicos. Sin duda, la asignatura de Estructura y función del cuerpo humano debe sentar las bases para la integración por parte del estudiante de este conocimiento anterior al estudio de la patología y los cuidados de enfermería.

Es necesario señalar, sin embargo, que este proyecto docente tiene puntos débiles sobre los que se está trabajando para poder ajustar las necesidades de formación de los estudiantes de enfermería con los recursos que la institución universitaria dispone. En primer lugar, deben considerarse las horas de trabajo del alumno fuera las aulas. Para poder desarrollar los contenidos de manera efectiva es imprescindible que el alumno dedique unas horas semanales a la asignatura destinadas básicamente a: ampliar apuntes, buscar información sobre algún tema propuesto, desarrollar contenidos, realizar los casos propuestos en clase, y preparar la materia para las clases prácticas, entre otros. Aunque este trabajo del alumno ha estado hasta ahora presente pero no contabilizado, la adaptación de los currículos formativos a los créditos ECTS considerará esta dedicación de manera relevante. Ésto ayudará también a evitar sobrecargas de trabajo en el alumno, además de ser un sistema más equitativo con el estudio realizado.

Otro punto débil a tratar es el hecho que desarrollar un proyecto docente de estas características exige un aumento considerable de las horas de dedicación del profesor. Así es, la introducción de metodologías que potencien la implicación del alumno en su aprendizaje conlleva destinar más tiempo para atenderlo de manera individualizada o grupal fuera de las aulas, en forma de tutorías o de seminarios. Por ello, el profesor observa como debe reducir el tiempo dedicado a preparar clases, diseñar ejercicios, realizar actividades administrativas propias del puesto, desarrollar y participar en proyectos académicos y profesionales, y por supuesto, el tiempo dedicado a la investigación y publicación científica. En las Escuelas Adscritas a la Universidad esta problemática se agrava, puesto que el profesor en dedicación exclusiva realiza, frecuentemente, más de una asignatura y posee una carga de trabajo, entre horas de docencia y horas destinadas a otras tareas académicas, superior a la exigible en los centros universitarios propios.

Aunque es fundamental en la universidad actual desarrollar proyectos docentes con metodologías que potencien la responsabilidad del alumno en su aprendizaje, y que orienten los contenidos hacia las competencias profesionales de manera mas concreta, es importante señalar que todo ello debe ir acompañado de una distribución equitativa de recursos personales y materiales, para que la integración de la universidad al espacio europeo de enseñanza superior sea verdaderamente efectiva y competitiva.

\section{AGRADECIMIENTOS}

A Montserrat Antonin, Jefa de Estudios en el curso 2002/03 y actual Coordinadora de Titulación de la Escola Universitaria d'Infermeria Gimbernat, y a Ángels Martín, Coordinadora de Titulación del centro en el curso académico 2002/03, por apostar 
por el cambio y la innovación en docencia, confiando en mi capacidad y experiencia profesional para idear y desarrollar este proyecto.

A los profesores Joan Carles Mas y Antoni Fogué por sus aportaciones y su adaptación al nuevo proyecto.

\section{BIBLIOGRAFÍA}

1. Arias JA, Cuadrado ML. Contenidos teóricos de las materias generales y especializadas en los planes de estudios de las diplomaturas de ciencias de la salud. Educación Médica 2003;6(4): 134-138.
2. Prada JA, Verástegui C, Velásquez R. Aportaciones del alumnado al estado actual de la calidad de la docencia en Anatomía. Educación Médica 2003;6(4):153-158.

3. Nebot-Cegarra J, Campillo M, Perez J. Influencia de la metodología docente en la adquisición rápida de conocimientos. Educación Médica 2003;6(1):37-43.

4. Palés J. La educación médica basada en las competencias finales del estudiante. Educación Médica 2001;4(1):1-2.

5. Falcó A. La nueva formación de profesionales: sobre la competencia profesional y la competencia del estudiante de enfermería. Educación Médica 2004;7(1):42-45.

6. Nolla M, Palés J, Gual A. Desarrollo de las competencias finales del estudiante. Educación Médica 2002;5(2):76-81. 